\title{
Determinant of Long-Term Quality of Life: Surgery in Breast Cancer Treatment
}

\author{
Thorsten Kühn
}

Frauenklinik, Städtische Kliniken Esslingen a.N., Germany

Surgery plays an important role in today's multidisciplinary approach to breast cancer. However, the extent of surgical procedures has decreased constantly over the last century since numerous trials have demonstrated the systemic character of the disease. Survival rates depend rather on tumor biology and a targeted systemic treatment than on the extent of tissue removal.

The long-term quality of life after breast cancer treatment is largely associated with the sequelae of surgical procedures. These relate to functional impairment as well as psychological distress. The introduction of breast-conserving therapy (BCT) has contributed significantly to an improvement in the quality of life of patients. In addition, surgeons have developed sophisticated techniques to preserve the breast or improve cosmetic outcome, even in unfavorable situations, by introducing oncoplastic surgery. A wide range of procedures have been introduced to allow primary or secondary reconstruction of the breast for those patients who still require mastectomy. With the sentinel node procedure, node-negative patients who do not require any further axillary treatment can be identified. However, full axillary dissection remains an important tool for node-positive patients, although its role in the subgroup of patients who are treated with BCT and radiotherapy is declining.

The objectives and the technical challenge for breast cancer surgery have changed significantly during the last few decades. The previous extensive standard operations intended to improve survival rates the surgical treatment of breast cancer have become extremely versatile and complex, although alone they contribute only modestly to survival in today's interdisciplinary treatment approach. Surgery is, however, an important tool to ensure locoregional control and preserve the quality of life with regards to function and psychological health. A large variety of procedures is needed to ensure these treatment goals.

Surgerical techniques for breast cancer are still in a process of evolution and improvement. These encompass new or more individualized indications and treatment concepts as well as advances in surgical technologies. This development is, however, associated with severe economic challenges.

In this issue of BREAST CARE four experienced breast surgeons present the most important issues that are currently being discussed in the field of breast cancer surgery. Individualized treatment approaches that reflect the specific tumor biology and treatment efficacy, as well as side effects and individual comorbidity, for each patient play an important role in today's oncology. How can evidence-based clinical guidelines be adapted to the individual situation of a patient, and how can the treatment objective of disease-free survival be weighed against quality of life in specific subgroups of patients? The article 'Extent of Primary Breast Cancer Surgery - Standards and Individual Concepts' by Heil and colleagues [1] examines these issues and reviews the existing literature for patient- and risk-adapted surgical strategies. This concerns margin status in BCT as well as the extent of skin/areola or nipple resection in mastectomy patients and the extent of lymph node surgery in women with node-negative or even node-positive disease. What role does age (e.g. very young patients, older women) or a family history of breast cancer play in the surgical decision making.

Thill [2] presents an excellent summary of new surgical options that are currently under evaluation. Reexcisions are a significant problem in the surgical management of breast cancer. Second interventions are associated with psychological problems for patients. They are also cost intensive and may lead to unfavorable cosmetic results. Several technical systems that can allow an intraoperative margin assessment are currently being evaluated. These techniques might reduce the reexcision rates in the future. Although the technical concept and preliminary results appear promising, further evaluation including a rigorous calculation of the cost effectiveness is required before these systems can be introduced into daily clinical routine. The same applies to improved technologies for the sentinel lymph node procedure (e.g. indigocyanin

\section{KARGER}

Fax +497614520714

Information@Karger.de

www.karger.com (c) 2012 S. Karger GmbH, Freiburg

$1661-3791 / 12 / 0075-0361 \$ 38.00 / 0$

Accessible online at:

www.karger.com/brc
Prof. Dr. Thorsten Kühn

Frauenklinik

Städtische Kliniken Esslingen a.N.

Hirschlandstr. 97

73730 Esslingen, Germany

T.Kuehn@klinikum-esslingen.de 
green fluorescence technique, hand-held SPECT for 3D visualization of the sentinel node, or the use of magnetic tracers) or the intraoperative histological assessment of sentinel lymph nodes by one-step nucleic acid amplification (OSNA). Radioguided occult lesion localization (ROLL), which may be combined with conventional wire localization, appears a promising technique to improve the localization of non-palpable lesions and reduce the resection volume. Ultrasound as a new surgical dissection tool requires an evidence-based demonstration of an improvement in well-defined endpoints (e.g. seroma formation and operation time) before standard techniques are modified and additional costs can be justified. The introduction of acellular dermal matrices (ADM) represents an important improvement in breast reconstruction. ADMs help provide an improved formation of the lower breast pole and the inframammary fold, especially in patients with insufficient autologous implant coverage. The high material costs, however, still restrict the wide use of ADM in clinical routine. In summary, the author concludes that promising new technologies are available to provide more individuality and less radicality in breast cancer surgery, but the relationship between efficacy and costs needs to be well balanced for each technology presented.

BRCA mutation carriers have a high life-long risk for developing ipsilateral and/or contralateral breast cancer. Prophylactic surgery can play an important role for the primary prevention of breast cancer. Individual patient counseling is mandatory for these patients. Recommendations require precise knowledge of the primary oncological risks and the effect of primary (surgery) or secondary (intensified surveillance) prevention strategies. Nestle-Krämling [3] gives an excellent overview of the numerous issues related to breast cancer surgery in BRCA mutation carriers. Besides the oncological aspects, this includes a wide range of surgical procedures and strategic considerations (timing of reconstruction, uni- or bilateral procedures).

In his article 'Breast Cancer Surgery and Financial Reimbursement' [4], Hoffmann explains the evolution of the German payment system. The classification for breast cancer interventions has been considerably improved through the new Operation and Treatment Procedure Classification (OPS) introduced in 2012. This new classification is based on a clear and complexity-based model developed by Hoffmann and Wallwiener that reflects the resource usage of different surgical procedures. The improvement of the classification system for surgical procedures does, however, not necessarily translate into the diagnosis-related group (DRG) that is the basis for the reimbursement for the clinical institutions. Therefore, the current payment system does not reflect the complexity of today's breast cancer surgery, a fact that must be considered when cost-intensive technical improvement is being discussed. On the other hand, lymph node surgery as a 'cost-triggering' procedure is constantly being reduced, rendering the economic situation for breast centers even more critical. In today's multidisciplinary approach, the expenses for a patient are rather defined by multiple clinical and psychological services and less dependent on specific surgical procedures. It appears doubtful if the current, exclusively surgery-driven payment system is suited to promoting clinical and surgical progress.

\section{Disclosure Statement}

The author declares no conflict of interests.

\section{References}

1 Heil J, Fuchs V, Golatta M, Wallwiener M, Domschke C, Sinn P, Lux M, Sohn C, Schütz F: Extent of primary breast cancer surgery - standards and individualized concepts. Breast Care 2012;7: DOI: 10.1159/000343976.
2 Thill M, Baumann K: New technologies in breast cancer surgery. Breast Care 2012;7: DOI: $10.1159 / 000343660$.

$3 \overline{\text { Nestle-Krämling C }}$, Kühn T: Role of breast surgery in BRCA mutation carriers. Breast Care 2012;7: DOI: $\underline{10.1159 / 000343717}$.
4 Hoffmann J, Wallwiener D: Breast cancer surgery and financial reimbursement in Germany. Breast Care 2012;7: DOI: $\underline{10.1159 / 000343294}$ 